\title{
In situ laser light scattering studies on the influence of kinetics on surface morphology during growth of $\ln _{0.2} \mathrm{Ga}_{0.8} \mathrm{As} / \mathrm{GaAs}$
}

\author{
M. U. González, a) Y. González, L. González, and M. Calleja \\ Instituto de Microelectrónica de Madrid, CNM-CSIC, C/Isaac Newton, 8. 28760-Tres Cantos, Madrid, \\ Spain \\ J. A. Sánchez-Gil \\ Instituto de Estructura de la Materia, CSIC, C/Serrano, 121, 28006-Madrid, Spain
}

(Received 26 May 2000; accepted for publication 4 December 2000)

\begin{abstract}
Using real-time in situ laser light scattering we study, in this work, the influence of growth kinetics on the initial development of the crosshatched morphology and its subsequent evolution. The crosshatched morphology is characteristic of relaxed low strained layers $(\varepsilon<2 \%)$ and has been traditionally related to the plastic relaxation process driven by generation and multiplication of dislocations. However we have observed that, if the growth rate is slow enough, the onset of crosshatch formation takes place at a layer thickness in which the dislocation formation and multiplication processes have not appeared yet. This reveals that the stress field generated by the small density of misfit dislocations formed by bending of the dislocations preexisting in the substrate is strong enough to affect the evolution of the growth front morphology. Our results also show that the starting point and evolution of this characteristic morphology depend on the growth rate in such a way that when the growth rate is lower the crosshatched morphology starts to develop at a smaller thickness and shows a faster evolution rate. (C) 2001 American Institute of Physics. [DOI: 10.1063/1.1345518]
\end{abstract}

\section{INTRODUCTION}

Strain, relaxation, and surface morphology of heteroepitaxial semiconductors is of great technological importance in two main aspects. First, the incorporation of strained layers in electronic devices such as advanced high electron mobility transistors or heterostructure lasers in order to improve their performance has become a current strategy. Second, the limited number of materials available as substrates makes necessary the growth of relaxed buffer layers to adapt the substrate lattice parameter to that of the device active layer. In both approaches, the layers usually present rough morphologies, which limit the subsequent device fabrication and degrade their specifications. ${ }^{1,2}$

It is well known that the surface of low-strained layers $(\varepsilon<2 \%)$ develops a crosshatched morphology once they exceed the thickness at which mechanisms of dislocation nucleation and multiplication start to work $\left(h_{p}\right) .^{3,4}$ The crosshatched morphology consists of ridges and grooves running parallel to $\langle 110\rangle$ directions with a well-defined average distance between consecutive ridges. This characteristic morphology could be caused, as generally reported in the literature, ${ }^{3-5}$ by variations in surface diffusion associated with inhomogeneous stress fields due to the presence of misfit dislocations in the layer.

The thickness where the dislocation multiplication process takes place in $\operatorname{In}_{x} \mathrm{Ga}_{1-x}$ As layers can be formulated as $K=h_{p} \varepsilon,{ }^{6}$ where $\varepsilon=\left(a_{p}-a_{0}\right) / a_{0}$ is the strain of the pseudomorphic layer, $a_{p}$ is the strained layer lattice parameter (which in the case of pseudomorphic layers is identical to the

${ }^{a)}$ Electronic mail: ujue@imm.cnm.csic.es substrate lattice parameter), and $a_{0}$ is the $\operatorname{In}_{x} \mathrm{Ga}_{1-x}$ As equilibrium lattice parameter. $K$ is an empirical constant that is equal to $0.8 \pm 0.1 \mathrm{~nm}$ for this material system, resulting in $h_{p}=56 \pm 7 \mathrm{~nm}$ for In content $x=0.2$.

Moreover, before reaching the layer thickness for dislocation nucleation and multiplication, misfit dislocations appear in the layer/substrate interface. Previously reported experimental results ${ }^{7}$ have demonstrated that when the lowstrained layers surpass the Matthews critical thickness $\left(h_{c}\right),{ }^{8}$ threading dislocations preexisting in the substrate begin to bend and misfit dislocation segments form at the layer/ substrate interface, as predicted by the Matthews relaxation process model. (Note: $h_{c} \cong 6 \mathrm{~nm}$ for In content $x=0.2$ ). However, no crosshatched morphology was clearly recognized during this first plastic relaxation process. ${ }^{4}$

Furthermore, some studies on the $\mathrm{SiGe} / \mathrm{Si}$ and InGaAs/ GaAs systems ${ }^{9-11}$ have shown that the surface can also roughen prior to misfit dislocation generation. This surface roughening is associated with an elastic stress relaxation process and depends strongly on the growth kinetics.

In this work we analyze the influence of the growth kinetics on the initial development of crosshatched morphologies and their subsequent evolution. An in situ laser light scattering (LLS) technique is used to monitor the evolution of the surface roughness in real time. LLS is a valuable technique for checking surface morphology evolution during epitaxial growth, ${ }^{3,12-15}$ since the LLS intensity is related to the mean surface roughness. ${ }^{16,17}$ The surfaces generated during epitaxial growth are highly smooth, thus allowing that the LLS technique is sensitive to surface features as small as nanometers in height. However, the Rayleigh limit restricts 
the lateral resolution to features larger than $\lambda / 2$.

Ex situ angle resolved light scattering (ARLS) and atomic force microscopy (AFM) characterization are used for a deeper insight in surface morphology after growth. $\mathrm{X}$-ray diffraction measurements are also made in order to assess the composition and strain state of the layers.

In particular, we have studied the dependence on the growth rate of the morphology evolution of $\mathrm{In}_{0.2} \mathrm{Ga}_{0.8} \mathrm{As} / \mathrm{GaAs}$ layers grown by molecular beam epitaxy (MBE). We have detected with in situ LLS measurements the existence of an initial kinetically controlled surface roughening consisting of ridges parallel to $\langle 110\rangle$ directions. These ridges are higher along [110] direction, the preferential surface diffusion direction in (001) oriented III-V semiconductor surfaces. ${ }^{18,19}$ This surface roughening takes place at a layer thickness $h_{r}$ well above Matthews critical thickness, but much earlier than dislocation multiplication effects appear, $h_{c}<h_{r}<h_{p}$. The value of $h_{r}$ depends on the growth rate. The subsequent morphology evolution and its relationship with growth kinetics and the strain relaxation process will also be discussed.

\section{EXPERIMENTAL PROCEDURE}

$\mathrm{In}_{x} \mathrm{Ga}_{1-x}$ As layers under study were grown by MBE. We have used on axis Si-doped GaAs (001) epiready substrates with conventional commercial specifications for threading dislocation density $\left(10^{4} \mathrm{~cm}^{-2}\right)$. After oxide thermal desorption, a $200 \mathrm{~nm}$ thick GaAs buffer layer was grown at a substrate temperature $T_{s}=580^{\circ} \mathrm{C}$. $\mathrm{In}_{x} \mathrm{Ga}_{1-x}$ As layers, with a nominal indium content $x=0.2$, were grown at $T_{s}=500{ }^{\circ} \mathrm{C}$ under an $\mathrm{As}_{4}$ beam equivalent pressure of 4 $\times 10^{-6}$ Torr using two different growth rates, namely 0.2 and $0.5 \mathrm{ML} / \mathrm{s}$.

In situ LLS measurements were carried out during MBE growth in order to monitor the morphology evolution dependence on the growth rate. The sample surface was illuminated with a $10 \mathrm{~mW} \mathrm{He}-\mathrm{Ne}$ laser $(\lambda=633 \mathrm{~nm})$ through a lateral viewport, at an angle of incidence $\theta_{i}=50^{\circ}$. The light scattered at an angle $\theta_{s}=0^{\circ}$ from the surface normal and at $\varphi_{s}=5^{\circ}$ away from the plane of incidence was collected with a teleobjective through a viewport that is facing the sample, and focused onto a silicon photodiode. We employ lock-in detection in order to reject spurious signals coming from the hot effusion cells and improve the signal-to-noise ratio.

Crosshatched morphologies produce a characteristic scattered light pattern consisting on bright lines strongly concentrated on $\langle 110\rangle$ directions. ${ }^{20}$ In order to follow the evolution of the growth front morphology in the directions where roughness develops during the crosshatch formation process, we carefully align before growth the [110] direction of the sample into the light scattering detection plane. With this geometry we can monitor in real time the evolution of the component along [110] of the surface power spectral density function (PSD) corresponding to a specific spatial frequency $|\mathbf{K}|=2 \pi / \lambda\left(\sin \theta_{s} \cos \varphi_{s}-\sin \theta_{i}\right)^{16,21}$ with a value of 7.6 $\mu \mathrm{m}^{-1}$ for our experimental setup. Accordingly, we can follow the evolution of the surface profile along [110] direction with maximum sensitivity for lateral length scales on the surface plane of $0.83 \mu \mathrm{m}$. We can also in situ rotate the sample to obtain information about the surface roughness along other directions.

The final surface topography has been assessed by means of ex situ ARLS measurements. In this case samples were illuminated with an $s$-polarized $\mathrm{He}-\mathrm{Ne}$ laser at two different angles of incidence, $\theta_{i}=0^{\circ}$ and $\theta_{i}=20^{\circ}$. Light scattered in the plane of incidence was collected with a silicon photodiode moving between scattering angles $\theta_{s}=-63^{\circ}$ and $\theta_{s}=69^{\circ}$. Several scans were taken with [110], [1 $\left.1 \overline{1} 0\right]$, and [100] surface directions contained in the plane of incidence. This procedure allows to retrieve the surface PSD components along those three directions for spatial frequencies in the range $|\mathbf{K}|=0.5-12 \mu \mathrm{m}^{-1}$ (lateral length scales $0.5-12.5$ $\mu \mathrm{m})$. By measuring the intensity scattered at a fixed angle $\theta_{s}=30^{\circ}$ during sample rotation under normal incidence we have also obtained the scattered intensity dependence on surface azimuth (AzLS), which is related to roughness anisotropy.

Surface morphology characterization for higher spatial frequencies (lateral length scales smaller than $0.5 \mu \mathrm{m}$ ) has been completed by AFM.

The relaxation degree of the $\operatorname{In}_{x} \mathrm{Ga}_{1-x}$ As samples was determined by x-ray diffraction (XRD) in an X'Pert MRD Philips diffractometer with four crystal Ge (220) as incident beam optic. The $(+-)$ Bragg arrangement for the (004) reflection and the $(\theta+\Phi)(\theta-\Phi)$ arrangement for (115) reflections were used. These four diffractograms were taken in both [110] and [1 $\overline{1} 0]$ directions for each sample. From the recorded data and using a dynamical simulation program we have obtained the alloy composition and the strain state in the $\operatorname{In}_{x} \mathrm{Ga}_{1-x}$ As samples. We would like to point out that, with a threading dislocation density in the substrate of $10^{4}$ $\mathrm{cm}^{-2}$, the degree of relaxation achieved in the layer by the Matthews relaxation process is so small that XRD measurements cannot detect any change in the lattice parameter of the layer. ${ }^{22}$ However, once the dislocation multiplication mechanism actuates, relaxation becomes enough to be detectable by this technique.

\section{RESULTS AND DISCUSSION}

Figure 1 shows the in situ recorded scattered light intensity during growth of two $200 \mathrm{~nm}$ thick $\mathrm{In}_{0.2} \mathrm{Ga}_{0.8}$ As layers. These layers were grown under the same conditions except for the growth rate $r_{g}$, which were 0.2 and $0.5 \mathrm{ML} / \mathrm{s}$. The LLS data were taken with the [110] direction of the sample contained in the detection plane. Window coating and small differences in optical alignment from run-to-run change the relationship between the relative in situ measured scattering intensities and relative roughness of each sample. In order to preserve a correlation between measured light and surface topography, in situ signals have been scaled considering the relation between the PSDs obtained from ex situ ARLS measurements. The scattered signal level just before InGaAs growth, which comes from the GaAs buffer layer, has been subtracted in order to fix the same initial value in the figure for both samples. 


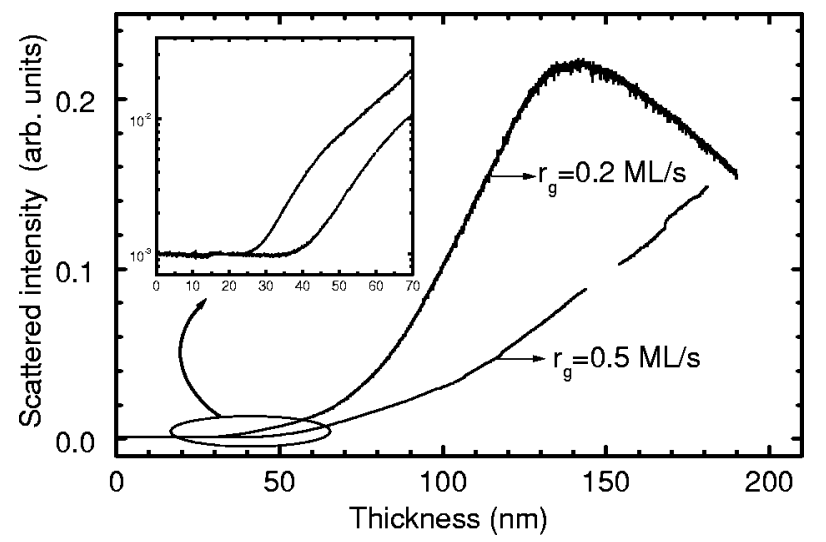

FIG. 1. In situ real-time LLS measurements of two $200 \mathrm{~nm}$ thick $\mathrm{In}_{0.2} \mathrm{Ga}_{0.8} \mathrm{As}$ layers grown by MBE at 0.5 and $0.2 \mathrm{ML} / \mathrm{s}$. Measurements have been taken with the [110] direction contained into the scattering detection plane. The inset shows a detail, in logarithmic scale, of the onset of LLS increase.

The results plotted in Fig. 1 show the same general behavior for both growth rates. From the beginning of the InGaAs layer growth to a certain thickness $h_{r}$, the LLS signal remains approximately constant, at a value that we will denote the ground level. At $h_{r}$ the signal begins to rise very fast. This increase in scattered light is related to a quick development of surface roughness along the [110] direction. We can assess if this roughening is associated with a crosshatched morphology by studying the azimuthal character of the surface topography. ${ }^{20}$ With this aim, we have also measured the intensity of the light scattered by a static surface in [110] or [100] directions for thickness of 100 and $200 \mathrm{~nm}$ (not shown in Fig. 1 for clarity). For these measurements the sample was quickly cooled down immediately after growth interruption, in order to freeze the morphology, and rotated for aligning the $[1 \overline{1} 0]$ or the [100] direction in the detection plane. Before growth was resumed, the LLS intensity measured with the [110] direction into the detection plane had the same value as just before growth interruption, demonstrating that surface topography remains constant during the surface quenching process.

For the [100] direction we always measured LLS signal values close to the ground level. On the other hand, in the $[1 \overline{1} 0]$ direction we detected a LLS signal increase with respect to the initial level, although it always remained well below the LLS value measured for [110] direction. We have also obtained that the LLS signals detected in both $\langle 110\rangle$ directions are sharply concentrated in those directions, and outside them the LLS signal abruptly drops getting values close to the ground level. This kind of azimuthal behavio clearly corresponds to a crosshatched-like morphology, ${ }^{20}$ showing that both samples have developed this characteristic roughness pattern as we expected in this low strained heteroepitaxial system.

Besides this general trend common to both samples, the in situ LLS measurements show clear differences in the crosshatch evolution for the two different growth rates explored (see Fig. 1). First, we observe a maximum in the scattered light intensity at a thickness of $140 \mathrm{~nm}$ for the layer grown at $0.2 \mathrm{ML} / \mathrm{s}$. Second, the morphology evolution is slower for the sample grown at $0.5 \mathrm{ML} / \mathrm{s}$. Finally, the thickness $h_{r}$ where the LLS signal begins to rise is growth rate dependent and takes place at a thickness much smaller than expected for crosshatch formation (critical thickness for dislocation multiplication $h_{p}=56 \mathrm{~nm}$ for $\operatorname{In}_{0.2} \mathrm{Ga}_{0.8} \mathrm{As}$ ) (see inset in Fig. 1).

The presence of the maximum for the layer grown at 0.2 $\mathrm{ML} / \mathrm{s}$ does not imply that the surface smoothens for higher thickness, but it is an artifact associated with the particular geometry we employ in our measurements. As our in situ experimental setup sensitivity is maximum for lateral length scales of $0.83 \mu \mathrm{m}$, we interpret this LLS intensity maximum as due to the evolution of the crosshatched morphology towards a topography whose main lateral distance is becoming much larger than $0.83 \mu \mathrm{m}$ along the [110] direction. ${ }^{3}$ This is the expected trend as the crosshatched morphology continuously evolves with thickness in the direction of higher ridges and larger average distances between consecutive ridges. Moreover, this maximum was not reached for the $0.5 \mathrm{ML} / \mathrm{s}$ grown layer even for thickness of $200 \mathrm{~nm}$, which is another indication of a slower morphology evolution for this sample.

The final surface morphology of these samples has been ex situ characterized by means of ARLS and AzLS measurements. Both measurements confirm the existence of a crosshatched morphology. From the ARLS measurements and employing scattering perturbation theory we have retrieved the surface PSDs of these $\operatorname{In}_{0.2} \mathrm{Ga}_{0.8}$ As layers. These PSDs allow us to obtain quantitative information about the crosshatch characteristic parameters, namely the average distance between consecutive ridges $d$ and its peak-to-valley height values $r$ for both $\langle 110\rangle$ directions. ${ }^{20,23}$ Table I presents the $d$ and $r$ values obtained from the calculated PSDs for the same samples as in Fig. 1. These data confirm that $r_{[110],[110]}(0.2$ $\mathrm{ML} / \mathrm{s})>r_{[110],[110]}(0.5 \mathrm{ML} / \mathrm{s})$ and $d_{[110]}(0.2 \mathrm{ML} / \mathrm{s})>$ $d_{[110]}(0.5 \mathrm{ML} / \mathrm{s})$, with $d_{[110]}(0.2 \mathrm{ML} / \mathrm{s}) \gg 0.83 \mu \mathrm{m}(0.83 \mu \mathrm{m}$ is the lateral length corresponding to the specific spatial frequency tuned in our in situ experimental setup), as we expected from our in situ recorded LLS measurements. So, these results evidence that the rate of crosshatch evolution is strongly dependent on the growth rate, being faster for lower growth rates.

The results plotted in Fig. 1 also show that the surface roughening onset thickness $h_{r}$ (LLS signal increase starting point), besides being growth rate dependent, occurs at a layer thickness much smaller than the critical for dislocation multiplication ( $h_{p}=56 \pm 7 \mathrm{~nm}$ for $\operatorname{In}_{0.2} \mathrm{Ga}_{0.8} \mathrm{As}$ ). According to previously published results, ${ }^{3,4}$ for a thickness below $h_{p}$ we would not expect a crosshatch development. In order to characterize the observed early surface roughening occurring for $h<h_{p}$, we have in situ recorded the LLS signal evolution during growth of another three $\operatorname{In}_{0.2} \mathrm{Ga}_{0.8}$ As layers. We used 0.2 and $0.5 \mathrm{ML} / \mathrm{s}$ as growth rates again. After growth they were characterized by XRD to study the role of relaxation in the initial surface roughening process. The XRD obtained values of In content $x$, in-plane residual strain $\varepsilon_{r}$, and the degree of relaxation $R$ are shown in Table I. $\varepsilon_{r}$ is defined as $\varepsilon_{r}=\left(a_{r}-a_{0}\right) / a_{0}$, where $a_{r}$ is the final lattice parameter of the InGaAs layer, and $R=\left(a_{r}-a_{s}\right) /\left(a_{0}-a_{s}\right)$, with $a_{s}$ the substrate lattice parameter. 
TABLE I. Roughness and relaxation characteristic parameters of the $\operatorname{In}_{x} \mathrm{Ga}_{1-x}$ As layers. $h$ and $r_{g}$ are thickness and growth rate of the layers. $x, \varepsilon_{r}$, and $R$ are experimental values of In content, in-plane residual strain and degree of relaxation respectively, obtained from XRD measurements. Average distances between ridges running along [110] direction and their height are represented by $d_{[110]}$ and $r_{[110]}$; the same parameters but for features aligned in the [110] direction are represented by $d_{[1 \overline{1} 0]}$ and $r_{[1 \overline{1} 0]}$. These values for the crosshatched morphologies are obtained through the PSDs calculated from ARLS measurements. Values marked with $*$ are obtained from AFM profiles. $d_{[110]}$ and $d_{[1 \overline{1} 0]}$ values have no sense in those cases since their value is associated with the density of misfit dislocations on the interface, and this depends on the particular area of the substrate measured.

\begin{tabular}{rcccccccc}
\hline \hline $\begin{array}{c}h \\
(\mathrm{~nm})\end{array}$ & $\begin{array}{c}r_{g} \\
(\mathrm{ML} / \mathrm{s})\end{array}$ & $x(\%)$ & $\begin{array}{c}\varepsilon_{r} \\
\left(\times 10^{3}\right)\end{array}$ & $\begin{array}{c}R \\
(\%)\end{array}$ & $\begin{array}{c}d_{[110]} \\
(\mu \mathrm{m})\end{array}$ & $\begin{array}{c}d_{[1 \overline{1} 0]} \\
(\mu \mathrm{m})\end{array}$ & $\begin{array}{c}r_{[110]} \\
(\mathrm{nm})\end{array}$ & $\begin{array}{c}r_{[1 \overline{1} 0]} \\
(\mathrm{nm})\end{array}$ \\
\hline 200 & 0.2 & 17 & $-5.4 \pm 0.4$ & $55 \pm 3$ & $2.0 \pm 0.2$ & $2.6 \pm 0.3$ & 10.2 & 2.8 \\
200 & 0.5 & 21 & $-5.0 \pm 0.5$ & $66 \pm 3$ & $0.8 \pm 0.1$ & $1.6 \pm 0.2$ & 7.3 & 1.7 \\
65 & 0.2 & 18 & $-12.6 \pm 0.1$ & $2 \pm 1$ & $0.8 \pm 0.1$ & $1.0 \pm 0.2$ & 2.8 & 1.4 \\
47 & 0.5 & 17 & $-12.2 \pm 0.1$ & $0 \pm 1$ & $\cdots$ & $\cdots$ & $1.1^{*}$ & $0.3^{*}$ \\
50 & 0.2 & 18 & $-12.9 \pm 0.1$ & $0 \pm 1$ & $\cdots$ & $\cdots$ & $1.8^{*}$ & $0.7^{*}$ \\
\hline \hline
\end{tabular}

Figure 2 shows the in situ recorded LLS measurements at [110] direction for the $0.2 \mathrm{ML} / \mathrm{s}$ grown $\operatorname{In}_{0.2} \mathrm{Ga}_{0.8}$ As layer. Its total thickness is close to the critical thickness for generation and multiplication of dislocations in the layer $(h=65$ $\mathrm{nm}, h_{p}=62 \pm 8 \mathrm{~nm}$; see x-ray results in Table I for actual composition values). We also show results for the $0.5 \mathrm{ML} / \mathrm{s}$ grown sample with a total thickness lower than $h_{p}(h=47.5$ $\mathrm{nm}, h_{p}=66 \pm 9 \mathrm{~nm}$ ), at which the LLS signal just started to take off. Also included are the final in situ recorded LLS intensity values for another $0.2 \mathrm{ML} / \mathrm{s}$ grown sample with thickness lower than critical $\left(h=50 \mathrm{~nm}, h_{p}=62 \pm 8 \mathrm{~nm}\right)$, but where LLS signal had already evolved. LLS data have been rescaled in the same way as data in Fig. 1.

From the LLS signal monitored at [110] and considering the discrete values obtained at the $[1 \overline{10}]$ direction (circles in Fig. 2), as well as the high directionality of roughness along those directions, we conclude that the $0.2 \mathrm{ML} / \mathrm{s}$ grown sample with a total thickness $h \approx h_{p}$ shows a well developed crosshatched morphology. This sample presents a small re-

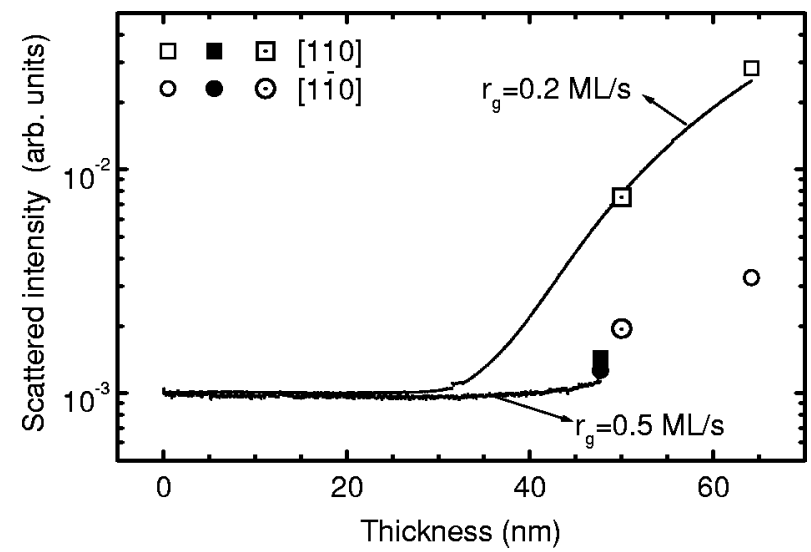

FIG. 2. In situ real-time LLS measurements of a $47 \mathrm{~nm}$ thick $\operatorname{In}_{0.2} \mathrm{Ga}_{0.8} \mathrm{As}$ layer grown at $0.5 \mathrm{ML} / \mathrm{s}$ and a $65 \mathrm{~nm}$ thick $\mathrm{In}_{0.2} \mathrm{Ga}_{0.8} \mathrm{As}$ layer grown at 0.2 $\mathrm{ML} / \mathrm{s}$. Measurements have been made in the same conditions as those in Fig. 1. LLS intensity obtained for [110] and [1 $\overline{1} 0]$ directions at final layer thickness, represented by squares and circles, respectively, are also included (open symbols: $0.2 \mathrm{ML} / \mathrm{s}$ grown sample, closed symbols: $0.5 \mathrm{ML} / \mathrm{s}$ grown sample). We have also inserted the final LLS intensity values obtained at [110] (dotted square) and [1 $1 \overline{1} 0]$ (dotted circle) directions for a $50 \mathrm{~nm}$ thick layer grown at $0.2 \mathrm{ML} / \mathrm{s}$. The previous LLS evolution of this latter sample is the same as that shown in this figure for the other $0.2 \mathrm{ML} / \mathrm{s}$ grown layer. laxation $R=2 \%$ (obtained by $\mathrm{x}$-ray diffraction measurements) as could be expected since the layer has just surpassed $h_{p}$.

Furthermore, LLS signal evolution and azimuthal behavior obtained from the two samples with $h \ll h_{p}$ also indicate that the increase of the LLS signal corresponds to crosshatch evolution even for thickness well below $h_{p}$. However, the XRD results show that both samples are fully strained. Summarizing, these results demonstrate that the crosshatch is formed before strain relaxation can be detected by $\mathrm{x}$-ray diffraction measurements.

Experimental results collected in Table I also show that both $200 \mathrm{~nm}$ thick layers (grown at 0.2 and $0.5 \mathrm{ML} / \mathrm{s}$ ) have the same strain state although their morphology evolution rate was completely different (see Fig. 1). This supports our assessment that it is the growth rate, and not a different relaxation rate, that determines the differences in roughness evolution.

AFM direct views of the morphology in the initial stages of surface roughening are shown in Figs. 3(a) and 3(b). The AFM image of Fig. 3(a) has been taken from the $47.5 \mathrm{~nm}$ thick layer grown at $0.5 \mathrm{ML} / \mathrm{s}$ (closed symbols in Fig. 2). Notice that the LLS signal just started to take off when the growth was stopped in this sample. The image of Fig. 3(b) corresponds to the $50 \mathrm{~nm}$ thick layer grown at $0.2 \mathrm{ML} / \mathrm{s}$ (open dotted symbols in Fig. 2). Both AFM viewgraphs evidence the presence on the surface of straight features running along both $\langle 110\rangle$ directions over a background consisting of elongated roughness along the $[1 \overline{1} 0]$ direction, the preferential surface diffusion direction in (001) oriented III-V MBE grown layers. ${ }^{18,19}$

The straight features along the [110] direction shown in Fig. 3(a) are $0.28 \mathrm{~nm}$ high, corresponding approximately to a monatomic step on the surface. These features are the footprint of $60^{\circ}$ misfit dislocation segments confined at the interface ${ }^{24}$ However, the straight features along the $[1 \overline{1} 0]$ are higher with an average value of $1.1 \mathrm{~nm}$ along a distance of 2 $\mu \mathrm{m}$. Although their extreme straightness unquestionably reveals that these features are related to the presence of $60^{\circ}$ misfit dislocation segments at the interface, in this case the surface morphology has evolved around them and incipient ridges as those of a crosshatched pattern are starting to form. 


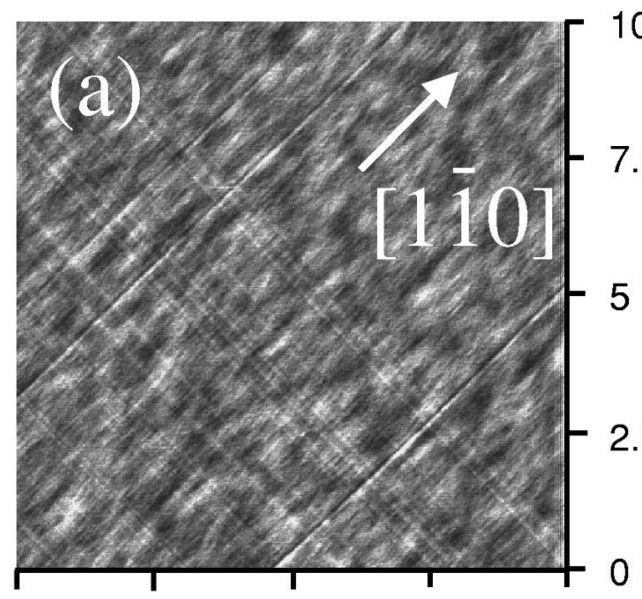

10
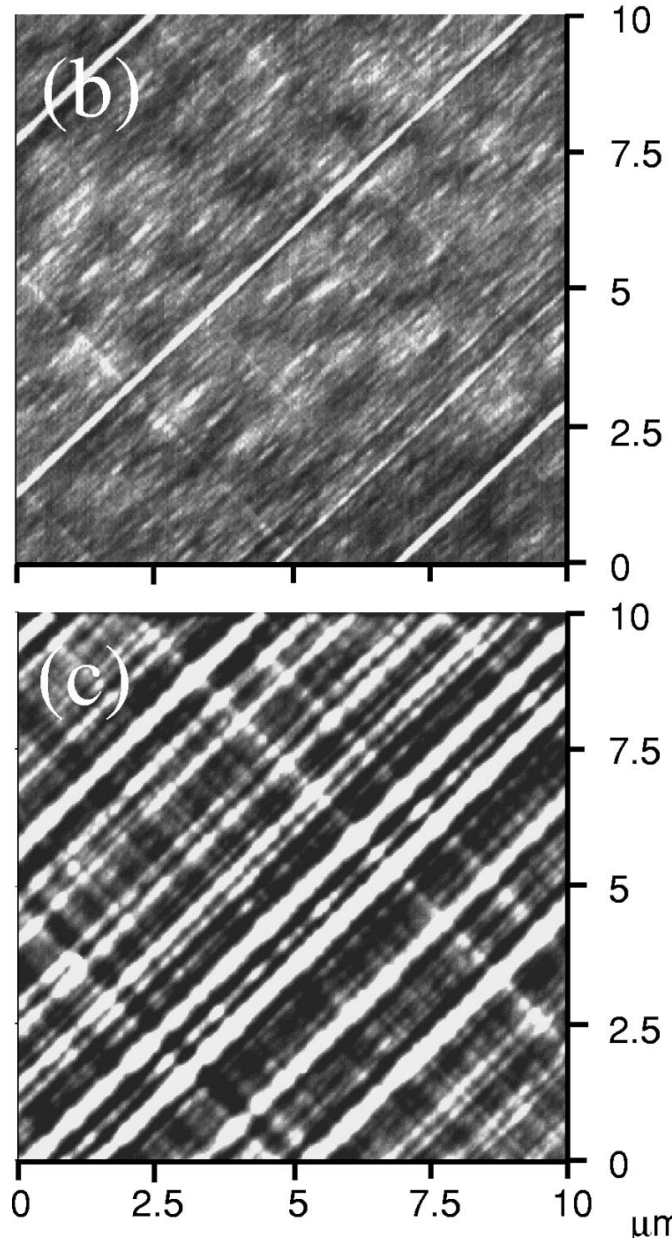

$\mu \mathrm{m}$

FIG. 3. AFM images of several $\operatorname{In}_{0.2} \mathrm{Ga}_{0.8}$ As layers: (a) viewgraph of the 47 $\mathrm{nm}$ thick layer grown at $0.5 \mathrm{ML} / \mathrm{s}$; (b) image of the $50 \mathrm{~nm}$ thick layer grown at $0.2 \mathrm{ML} / \mathrm{s}$; (c) micrograph of the $65 \mathrm{~nm}$ thick layer grown at $0.2 \mathrm{ML} / \mathrm{s}$. The LLS behavior of these samples is shown in Fig. 2. All images are represented with the same height scale.

Taking into account that for the sensitivity of the XRD technique this sample is fully strained, and that $h_{c}<h<h_{p}$, we can assume that the $60^{\circ}$ misfit dislocations have been formed through the Matthews relaxation process, in which the threading dislocations preexisting in the substrate bend to form misfit segments at the interface. ${ }^{8}$

On the other hand, morphology shown in Fig. 3(b) corresponds to a further step in surface topography evolution, still with $h<h_{p}$. Here, the straight features in both directions can also be linked to the existence of misfit dislocation segments at the interface and in both cases ridges have begun to develop. We obtain height values around $0.7 \mathrm{~nm}$ for ridges aligned along [110] direction. Along [110], ridges are higher with an average height of $1.8 \mathrm{~nm}$ over a distance of $2.5 \mu \mathrm{m}$. Although the thickness for both samples in Figs. 3(a) and 3 (b) is very similar, the roughness is more developed in the $0.2 \mathrm{ML} / \mathrm{s}$ grown sample, as we had already seen from the in situ LLS measurements. Ripple heights for these samples, as obtained from the AFM measurements, are also included in Table I.

For both samples, the variation in the density of misfit dislocations along [110] and [1 $1 \overline{1} 0]$ is not related to different stages in the relaxation process, but just depends on the particular type and region of the substrate that we are viewing, as has been previously demonstrated. ${ }^{7}$

If the crosshatched ridges begin to develop at this initial stage of the relaxation process, then we can expect that when the multiplication of dislocation process takes place, welldeveloped ridges already exist in the surface, in a density imposed by the particular defect distribution of the substrate region implied.

Figure 3(c) corresponds to an AFM view of the $0.2 \mathrm{ML} / \mathrm{s}$ grown sample with total thickness $h \approx h_{p}$ and $R=2 \%$ (open symbols in Fig. 2). This topography shows a clear crosshatched morphology with well-defined ridges running along both $\langle 110\rangle$ directions. Most of the ridges along the [110] direction have a peak-to-valley distance $r$ around $2 \mathrm{~nm}$. However, some ridges appear with $r$ values up to $4.5 \mathrm{~nm}$. These ridges could be those that started to form in the first stage of roughening of the layer [Figs. 3(a) and 3(b)].

Our results show that if the growth rate is slow enough, incipient ridges as those of the crosshatched morphology appear along $\langle 110\rangle$ directions [Figs. 3(a) and 3(b)] at a layer thickness well below the critical thickness for dislocation multiplication, considered traditionally as the starting point for crosshatch formation. At the layer thickness for ridge formation, only the Matthews relaxation process has taken place. At this stage no dislocations have nucleated yet at the surface and there are highly separated misfit dislocations at the substrate/layer interface. The stress field produced by this low density of misfit dislocations confined at the interface is strong enough to produce a local increase of the growth rate in those sites where strain level is reduced. This local increase will be controlled by surface diffusion and, accordingly, the lower the growth rate, the higher will be the stress field effects on the growth front morphology, in total agreement with our experimental results. Moreover, these effects are more evident along the $[1 \overline{1} 0]$ direction, which is the preferential surface diffusion direction in (001) oriented III-V semiconductor surfaces. ${ }^{18,19}$

\section{SUMMARY AND CONCLUSION}

We have studied in situ and in real time the evolution of surface morphology during MBE growth of $\mathrm{In}_{0.2} \mathrm{Ga}_{0.8} \mathrm{As}$ on GaAs (001) substrates by LLS. After growth, surface mor- 
phology has been characterized by ex situ ARLS and AFM. Relaxation and composition of the grown layers have been assessed by $\mathrm{x}$-ray diffraction.

Our results show that for 0.2 and $0.5 \mathrm{ML} / \mathrm{s}$ growth rates a crosshatched surface morphology starts to develop at a layer thickness much smaller than the critical thickness for dislocation multiplication process $\left(h_{p}\right)$. We have observed that the onset of LLS signal, directly related to surface roughness, depends on growth rate. The subsequent evolution of the crosshatched topography is also controlled by the growth rate.

From our experimental results we can conclude that the presence of misfit dislocations at the InGaAs/GaAs interface corresponding to the Matthews relaxation process produces stress fields at the surface strong enough to affect the morphology evolution of the growth front. If the rate of arrival of group III atoms is low enough, they have time, before incorporation into the lattice, to reach surface areas where strain level has been reduced by the presence of misfit dislocations. In that case the stress field is revealed by the appearance of ridges at the surface. This process is enhanced in the preferential surface diffusion direction $([1 \overline{1} 0]$ for $(001)$ oriented III-V semiconductors).

Our results show that the surface roughness evolution at thickness below $h_{p}$ has to be taken into account for a global understanding of the crosshatch formation process.

\section{ACKNOWLEDGMENTS}

The authors wish to acknowledge the Spanish "CICYT', for financial support under Project No. TIC991035-C02. Two authors (M.U.G. and M.C.) thank the Consejería de Educación y Cultura de la Comunidad de Madrid for financial support, which also supports in part the work of J. A. Sánchez-Gil through Project No. 07M/0040/1999.
${ }^{1}$ N. Rorsman, C. Karlsson, S. M. Wang, H. Zirath, and T. G. Andersson, Electron. Lett. 31, 1292 (1995).

${ }^{2}$ I. J. Fritz, B. E. Hammons, A. J. Howard, T. M. Brennan, and J. A. Olsen, Appl. Phys. Lett. 62, 919 (1993).

${ }^{3}$ T. Pinnington, C. Lavoie, T. Tiedje, B. Haveman, and E. Nodwell, Phys. Rev. Lett. 79, 1698 (1997).

${ }^{4}$ R. Beanland, M. Aindow, T. B. Joyce, P. Kidd, M. Lourenço, and P. Goodhew, J. Cryst. Growth 149, 1 (1995).

${ }^{5}$ E. A. Fitzgerald, Y.-H. Xie, D. Monroe, P. J. Silverman, J. M. Kuo, A. R. Kortan, F. A. Thiel, and B. E. Weir, J. Vac. Sci. Technol. B 10, 1807 (1992).

${ }^{6}$ D. J. Dunstan, P. Kidd, R. Beanland, A. Sacedón, E. Calleja, L. González, Y. González, and F. J. Pacheco, Mater. Sci. Technol. 12, 181 (1996).

${ }^{7}$ S. J. Barnett et al., J. Phys. D: Appl. Phys. 28, A17 (1995).

${ }^{8}$ J. W. Matthews and A. E. Blakeslee, J. Cryst. Growth 29, 273 (1975).

${ }^{9}$ A. G. Cullis, D. J. Robbins, A. J. Pidduck, and P. W. Smith, J. Cryst. Growth 123, 333 (1992).

${ }^{10}$ M. Albrecht, S. Christiansen, J. Michler, W. Dorsch, H. P. Strunk, P. O. Hansson, and E. Bauser, Appl. Phys. Lett. 67, 1232 (1995).

${ }^{11}$ F. Peiró, A. Cornet, M. Beck, and M. A. Py, Appl. Phys. Lett. 74, 3818 (1999)

${ }^{12}$ G. W. Smith, A. J. Pidduck, C. R. Whitehouse, J. L. Glasper, and J. Spowart, J. Cryst. Growth 127, 966 (1993).

${ }^{13}$ A. R. Boyd, T. B. Joyce, and R. Beanland, J. Cryst. Growth 164, 51 (1996).

${ }^{14}$ U. Rossow, N. Dietz, K. J. Bachmann, and D. E. Aspnes, J. Vac. Sci. Technol. B 14, 3040 (1996).

${ }^{15}$ E. Chason, M. B. Sinclair, J. A. Floro, J. A. Hunter, and R. Q. Hwang, Appl. Phys. Lett. 72, 3276 (1998).

${ }^{16} \mathrm{~J}$. M. Bennett and L. Mattson, Introduction to Surface Roughness and Scattering (Optical Society of America, Washington, DC, 1999).

${ }^{17}$ J. A. Ogilvy, Theory of Wave Scattering from Rough Surfaces (Hilguer, Bristol, 1991).

${ }^{18}$ M. D. Pashley, Phys. Rev. B 40, 10481 (1989).

${ }^{19}$ K. Shiraishi, Appl. Phys. Lett. 60, 1363 (1992).

${ }^{20}$ M. U. González, J. A. Sánchez-Gil, Y. González, L. González, and E. R. Méndez, J. Vac. Sci. Technol. B 18, 1980 (2000).

${ }^{21}$ E. L. Church, H. A. Jekinson, and J. M. Zavada, Opt. Eng. 16, 360 (1977).

${ }^{22}$ P. Kidd, P. F. Fewster, N. L. Andrew, and D. J. Dunstan, Inst. Phys. Conf. Ser. 134, 585 (1993).

${ }^{23}$ M. U. González, J. A. Sánchez-Gil, Y. González, L. González, R. Garcaía, A. San Paulo, and J. M. García, J. Cryst. Growth 201/202, 137 (1999).

${ }^{24}$ F. R. N. Nabarro, Theory of Crystal Dislocations (Dover, New York, 1987). 